\title{
Die Vielfalt der Biophysikalischen Informations-Therapie (BIT) - einfach, sicher, hilfreich
}

\section{Bodo Köhler \\ Facharzt für Innere Medizin, Naturheilverfahren und Homöopathie, Freiburg, Deutschland}

BIT verkörpert das älteste Heilprinzip, das es gibt: Der Organismus wird mit sich selbst und all seinen Problemen in Resonanz gebracht. Es wird ihm sozusagen der Spiegel vorgehalten, woraufhin eine Neubewertung und erneute Auseinandersetzung mit unerledigten Dingen aus der Vergangenheit erfolgen kann. Dadurch werden Heilungsprozesse aktiviert, wodurch chronische Krankheitszustände beendet und eine neue Ordnung induziert werden können.

Wir leben im Informationszeitalter. Jeder kommuniziert jeden Tag unzählige Male mit verschiedenen Personen. Das ist Selbstverständlichkeit, und wir denken nicht darüber nach. Genau das Gleiche machen unsere Zellen und Organe ständig, allerdings mit einer viel höheren Übertragungsrate an Bit. Von der Qualität der Informationsübertragung hängt es ab, ob die funktionellen Vorgänge im Organismus mit grosser Präzision ablaufen können.

Werden Medikamente zugeführt, verändern sie durch ihren spezifischen Informationsgehalt die Informationsübertragung im Organismus. Das kann störend sein (Nebenwirkungen) oder unterstützend wirken, sodass auf diese Weise Symptome verschwinden können. Eine andere Erklärung der Medikamentenwirkung ist wissenschaftlich nicht haltbar. Der frühere Ansatz, dass sich die Chemie des Körpers verändern würde, muss fallengelassen werden, unter anderem deshalb, weil die gleichen Effekte auch dann eintreten, wenn ganz unterschiedliche Eintrittspforten gewählt werden.

Vor dem Hintergrund, dass die Masse nur einen Milliardsten Teil der Realität ausmacht, wir also fast ausschliesslich energetische Phänomene wahrnehmen, die durch spezifische Informationen gesteuert werden, liegt es auf der Hand, die Bedeutung der Information an die erste Stelle zu rücken.
Genau das macht die BIT. Statt sich mit Folgephänomenen der Materie zu beschäftigen, wird hier direkt an der Ursache mit der alles bewirkenden Information gearbeitet, und zwar ohne einen Umweg über Medikamente.

In einem Zeitraum von über drei Jahrzehnten hat sich durch unzählige praktische Anwendungen ein Erfahrungsschatz herauskristallisiert, der es ermöglichte, die Therapievarianten der BIT immer mehr zu verfeinern und auszubauen. Hinzu kamen völlig neue Erkenntnisse über die Lebensprozesse. Trotz allem bleibt das Prinzip der BIT einfach und überschaubar. Daran ändert auch die Vielfalt der heute angebotenen Gerätetypen nichts, denn dies ist nötig und richtig, um der Individualität aller Therapeuten Rechnung zu tragen und grossen Raum für Kreativität zu lassen.

Die BIT der heutigen Zeit beruht auf vier Säulen:

- Zelle-und-Milieu-Revitalisierung (ZMR; Zellstoffwechsel-Säuren/Basen)

- exogene BIT (Farb-/Ton-Therapie)

- Matrix-Regenerations-Therapie (MRT)

- endogene (klassische) BIT (mit patienteneigenen Signalen) Selbstverständlich ist vor jeder Behandlung eine Diagnose erforderlich. Diese wird völlig im Sinne der Naturheilkunde individuell und ganzheitlich erhoben und besteht deshalb aus vier Punkten:

1. Stoffwechsellage (anabol/katabol); Zustand des Milieus (sauer/basisch)

2. Konstitution (genetischer Schwachpunkt)

3. Dauerstressfaktoren (mental, emotional, somatisch)

4. Auslöser (vordergründige «Ursache»)

Der erfahrene Therapeut orientiert sich jeweils am «Leitsymptom» (das gewöhnlich der schulmedizinischen Diagnose entspricht). Hier wird jedoch nicht behandelt, sondern dieses als Referenz für den Therapiefortschritt benutzt. Verschwindet dies, ohne dass an dieser Stelle behandelt wurde, zeigt das den Erfolg einer kausalen Therapie an.

Es wird primär versucht, die tiefer liegende Ursache für die vorliegende Stoffwechselentgleisung $\mathrm{zu}$ finden, und zwar auf allen Ebenen des Seins. Die Suche wird nach

\section{Agenda UNION}

Vorstandssitzung UNION

Vorstandssitzung UNION

Vorstandssitzung UNION
Donnerstag, 10. November 2011 (Nachmittag); Bern

Donnerstag, 22. Dezember 2011 (Nachmittag/Abend); Bern

Donnerstag, 26. Januar 2012 (Nachmittag); Bern 
den Regeln der «Lebenskonformen Medizin» auf PsychoEbene, Stoffwechsel-Ebene und Matrix-Ebene durchgeführt und dort auch behandelt (siehe Köhler B: Die Grundlagen des Lebens). Das ist der Grund, warum sich inzwischen vier Säulen der Therapie herausgebildet haben. Der versierte Behandler kann damit ganz individuelle und ausserdem synergistisch wirkende Therapiekonzepte erstellen.

Zellstoffwechsel, Säuren/Basen-Status sowie die Regulationsfähigkeit von beiden dienen als Referenz für die weitere Behandlung. Diese Parameter sollten sich schrittweise bis zur Norm bessern. Das Ziel ist aber nicht, einen bestimmten Normwert zu erreichen, sondern primär die körpereigene Regulationsfähigkeit wiederherzustellen. Darunter ist die sogenannte "positive Reizantwort» $\mathrm{zu}$ verstehen. Sobald der Organismus dazu wieder in der Lage ist, läuft die Heilung autonom ab.

Damit eine Therapie überhaupt eine bestimmte Wirkung zeigen kann, müssen zunächst die notwendigen Heilungsvoraussetzungen geschaffen werden. Das umfasst die positive Ausrichtung der Psyche sowie die Normalisierung des Zellstoffwechsels und des Säuren/Basen-Haushaltes, weil nur dann der Organismus optimal reagieren kann. Davon ist die Effizienz jeder Therapie abhängig. Dazu eignet sich am besten das ZMR-Gerät, mit dem Stoffwechsel und gleichzeitig Säuren/Basen-Haushalt vollautomatisch reguliert und über die Farben Einfluss auf die Psyche genommen werden kann. Gleichzeitig können aufgrund der ermittelten Werte ganz gezielte Ernährungsempfehlungen gegeben werden.

Die Belastungen der Matrix (Dauerstressfaktoren) werden gewöhnlich nach einer festgelegten Abfolge ermittelt. Zunächst wird auf Schwermetallbelastungen und dann auf Wohngifte sowie Impfbelastungen getestet. Über Nosoden-Tests können bakterielle und virale Restentzündungen aufgedeckt werden, wobei hier Kinesiologie, Biotensor, Vegatest, bioelektronische Funktionsdiagnose (BFD) oder Elektroakupunktur nach Voll (EAV) zum Einsatz kommen. Die notwendige Ausleitung kann dann gezielt mit der endogenen BIT über ein «Aufmerksamkeitssignal» oder unspezifisch in mehreren Sitzungen mit der MRT erfolgen.

Auch vor der MRT sollten Zellstoffwechsel und Säuren/ Basen-Haushalt einreguliert werden, was am effektivsten mit dem ZMR-Gerät gelingt. Parallel zu den Sitzungen werden andere Behandlungen durchgeführt, wobei die Konstitutionstherapie mit Farben/Tönen/Edelsteinen Vorrang hat. Schliesslich sind wir auf ein gut funktionierendes Immunsystem und eine ausreichend hohe Energielage (Zellpotenzial) angewiesen, wenn wir Informationen in das System einspeisen. Die Voraussetzungen lassen sich durch die Konstitutionsbehandlung wesentlich verbes- sern. Genetische Schwachpunkte können nach den Regeln der TCM damit deutlich aufgebessert werden.

Es sollte das Anliegen jedes Therapeuten sein, zunächst den (dynamischen) Ordnungsgrad im Gewebe zu erhöhen und erst dann mit gezielten endogenen Signalen Herde oder Ähnliches zu behandeln.

Schwer chronisch kranke Patienten erfordern ein intensives Therapieregime. Nach mehrmaligem Ausgleich der Zellstoffwechsel- und Säuren/Basen-Regulation im Laufe von wenigen Wochen, einigen MRT-Sitzungen und individueller Konstitutionstherapie werden Störfelder dann mit der endogenen BIT behandelt. Dazu wird die Information mit Hautelektroden direkt am Herd abgegriffen und unverändert (!) symmetrisch zur Gegenseite geleitet (oder an eine andere gesunde Stelle im Organismus), um hier ein «Aufmerksamkeitssignal» zu setzen. Die Antwort kann zum Teil sehr heftig und mit den Zeichen einer akuten Entzündungsheilreaktion ablaufen, worauf der Patient vorbereitet werden sollte. Eine solche Behandlung wird im Wochenabstand bis zu zweimal wiederholt.

Die Behandlung eines schwer kranken Patienten kann mehrere Wochen in Anspruch nehmen, wobei hier ganz besonders die Arndt-Schulz'sche Regel Berücksichtigung finden sollte. Deshalb gilt auch hier: Weniger ist oft mehr.

Jeder Patient hat seine eigene Krankengeschichte und seinen ganz persönlichen Verlauf. Deshalb ist es selbstverständlich, dass nur eine ganz individuell angepasste Therapie die besten Resultate zeigen wird. Trotzdem kann ein Stufenschema hilfreich sein, das bei jedem Patienten entsprechend modifiziert Anwendung findet:

1. Heilungsvoraussetzungen schaffen: ZMR unterstützt die positive Ausrichtung der Psyche und stellt die Regulationsfähigkeit von Zellstoffwechsel und Säuren/BasenHaushalt wieder her.

2. Konstitutionstherapie: Farben/Töne/Edelsteine beeinflussen den genetisch determinierten Schwachpunkt und verbessern ihn funktionell nach den Regeln der TCM.

3. Entlastungstherapie: MRT und/oder endogene BIT zur Reinigung der Matrix von Toxinen, Schlacken, Störfeldern sowie viralen und bakteriellen Belastungen.

4. Unterstützung des Immunsystems orthomolekular: Vitamine, Mineralstoffe, Organopräparate oder Ähnliches.

Im Rahmen dieses Ablaufs werden die verschiedenen BIT-Varianten zielgerichtet eingesetzt. Die Auswahl richtet sich nach dem Schweregrad. Unverzichtbar ist jedoch Punkt 1. Denn ohne zunächst die notwendigen Heilungsvoraussetzungen zu schaffen, d.h. vorhandene Blockaden auf allen Regulationsebenen zu lösen, wird kein durchschlagender Erfolg zu erwarten sein. Deshalb nimmt die ZMR mit dem entsprechenden Gerät eine zentrale Stellung 


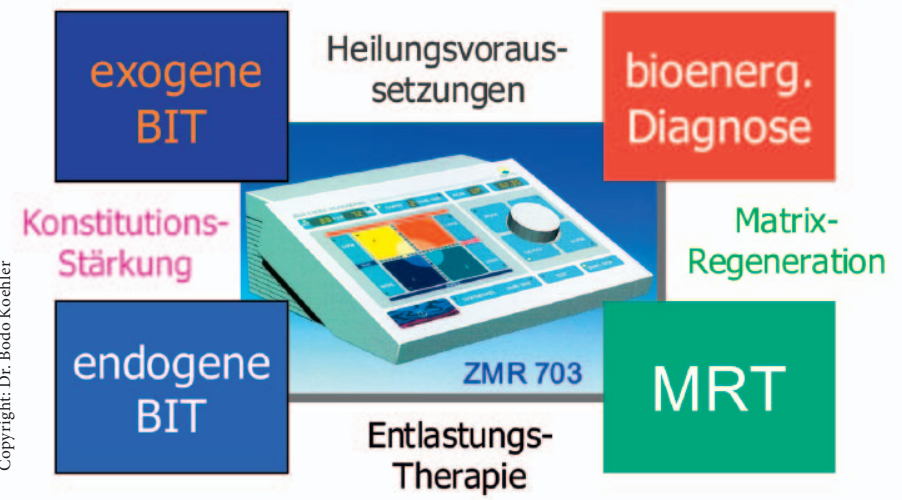

Abb. 1. Stellenwert der Zelle-und-Milieu-Revitalisierung in Verbindung mit anderen BIT-Methoden.

ein, um die sich die anderen Therapieformen gruppieren (Abb. 1). Sie ist immer der erste Schritt, bevor weitere Anwendungen folgen. Jedoch auch Methoden ausserhalb der BIT, z.B. Akupunktur, Neuraltherapie, Homöopathie usw., werden effektiver nach einer ZMR-Anwendung.

Das oben geschilderte Vorgehen unter Ausschöpfung der vielfältigen Möglichkeiten, welche die BIT bietet, kann zu ganz erstaunlichen Heilerfolgen führen, die mit anderen $\mathrm{Me}-$ thoden in der Regel nicht erreichbar sind. Hinzu kommt die leichte Anwendbarkeit, teilweise im Delegationsverfahren.

BIT kann in vielen Fällen mit gutem Erfolg ganz allein eingesetzt werden. Sie eignet sich jedoch auch hervorragend zur Begleitbehandlung anderer Methoden bis hin zur Krebstherapie. Schwerwiegende Nebenwirkungen belastender Medikamente oder einer Bestrahlung können abgeschwächt und das Allgemeinbefinden verbessert werden. Auch zur OP-Vorbereitung lassen sich sehr gute Wirkungen erzielen, die sich dann in kurzen Heilzeiten und verkürzten Krankenhausaufenthalten positiv niederschlagen.

Doch selbst nach einer OP oder einem zahnchirurgischen Eingriff bietet sich die Möglichkeit, den Heilungsvorgang aktiv $\mathrm{zu}$ unterstützen. Dazu wird etwas von dem OP-Material benötigt, z.B. ein kleiner Tumorrest, ein extrahierter Zahn oder Ähnliches.

Es wird immer wieder die Frage gestellt, für welche Erkrankungen sich die BIT besonders eignet. Eine solche Frage ist leider völlig unangebracht. Sie entspringt schulmedizinischem Denken, das kranke Menschen in Gruppen einteilt. Wir behandeln jedoch im Sinne der Naturheilkunde völlig individuell. Deshalb hat jeder Patient auch seine ganz persönliche Chance, gesund zu werden und den Weg zu gehen, der für ihn das Optimum darstellt.
BIT kann mit Erfolg bei jedem Menschen eingesetzt werden, ganz gleich, unter welchen Symptomen er leidet. Erst im Verlauf der Behandlung zeigt sich, wie gut er darauf anspricht. Es sollte an dieser Stelle nochmals betont werden, dass BIT nur dann die grössten Erfolge verbuchen kann, wenn tatsächlich die Besonderheiten, die bei jedem Patienten vorliegen, zur Grundlage der Therapie gemacht werden. Wertvolle Ressourcen werden verschenkt, wenn pauschal nach Indikationen behandelt wird, so wie es in der Schulmedizin üblich ist.

Abschliessend soll darauf hingewiesen werden, dass auch die BIT - trotz ihrer beeindruckenden Therapieresultate - kein Allheilmittel darstellt. Ein solches wird es nie geben. Wir werden mit jeder Methode, ganz gleich welcher, immer wieder Misserfolge erleben. Der Grund liegt einfach darin, dass der Patient ganz allein entscheidet, ob er gesund werden kann (und will!) oder nicht. Heilung ist ein aktiver Prozess, den der Patient selbst vollbringen muss. Ein aussergewöhnliches Therapieverfahren wie die BIT kann ihn dabei sehr gut unterstützen und neben den Grundvoraussetzungen, nämlich der Wiederherstellung autonomer Regulationsprozesse auf allen Ebenen, für eine durchgreifende Entlastung der verschiedenen Gewebe sorgen. Damit ist allerdings die Grenze erreicht. Den letzten Schritt muss der Patient selbst gehen.

Um die BIT sicher anwenden zu können, ist eine besondere Ausbildung erforderlich. Diese wird firmenneutral durch die Internationale Ärzte-Gesellschaft für Biophysikalische Informations-Therapie e.V. durchgeführt (www.bit-org.de).

\section{Kontakt}

Dr. med. Bodo Köhler

Facharzt für Innere Medizin, Naturheilverfahren und Homöopathie Brombergstrasse 33, 79102 Freiburg, Deutschland

\section{Literatur}

Bischof M: Biophotonen - das Licht in unseren Zellen. Frankfurt/M., Verlag 2001, 1995.

Kaucher E: Gegenwart und Zukunft der Menschheit - Neues Denken in der Medizin. Vortragsmanuskript Salveo-Kongress. Leipzig, 1995.

Kiene H: Komplementärmedizin - Schulmedizin. Der Wissenschaftsstreit am Ende des 20. Jahrhunderts, ed 2. Stuttgart, Schattauer, 1996.

Köhler B: Biophysikalische Informations-Therapie. Niebüll, Videel, 2002.

Köhler B: Das praktische Arbeitsbuch zur BIT. Hochheim, Co'Med, 1997.

Köhler B: Die Grundlagen des Lebens - Stoffwechsel und Ernährung. Niebüll, Videel, 2001.

Popp FA, et al: Electromagnetic Bio-information. München, Urban und Schwarzenberg, 1989.

Rubbia C: Nobelpreis 1984 für den experimentellen Nachweis der der Materie übergeordneten Wechselwirkungsquanten, welche die Struktur der Materie steuern.

Schole J, Lutz W: Regulationskrankheiten. Niebüll, Videel, 2001.

Smith CW, Best S: Electromagnetic Man. London, J.M. Dent \& Sons, 1989.

Stühmer R: Körper und Geist. München, Universitas, 1997.

Zycha H: Organon der Ganzheit. Heidelberg, Haug, 1996. 